\title{
Eczema-like plaques secondary to enoxaparin misdiagnosed as DRESS-syndrome
}

\section{Kamar Belhareth', Rim Sahnoun², Sonia Boudaya', Kamilia Ksouda', Emna Bahloul', Serria Hammemi ${ }^{2}$, Khaled Zghal' ${ }^{2}$, Hamida Turki ${ }^{1}$}

${ }^{1}$ Dermatology Department, Hedi Chaker Hospital, Sfax, Tunisia, ${ }^{2}$ Laboratory of Pharmacology, Faculty of Medicine, Sfax, Tunisia

Corresponding author: Kamar Belhareth, MD, E-mail: kmar.belhareth@gmail.com

\begin{abstract}
Low-molecular-weight heparins may cause adverse skin reactions, especially eczematous eruptions. A 60-year-old woman with a history of mitral valve replacement and frontal hemorrhagic stroke, who had been treated with carbamazepine and enoxaparin for 2 months, consulted our department for a sudden onset of generalized pruritic rash. There was facial edema, cheilitis, and a rash of eczematous lesions electively localized to the site of enoxaparin injections. Blood analysis showed eosinophilia $=2500 \mathrm{e} / \mathrm{mm} 3$, gamma GT $=526$ and lipasemia $=126$. Initially we suspected the diagnosis of carbamazepine induced DRESS syndrome. Then the final diagnosis was generalized eczema on heparin in view of the exclusive localization of the lesions at the sites of enoxaparin injections, the rapid resolution of the eruption and the result of biopsy and allergological investigation. Dermatologic effects of heparins commonly used in medical and surgical practice are numerous, and clinical pictures can be misleading, as in our case.
\end{abstract}

Key words: Heparin; DRESS; Drug reaction; Eczema

\section{INTRODUCTION}

Low-molecular-weight heparins (LMWHs) are commonly used for the prophylaxis and treatment of venous thromboembolism. However, they can cause adverse skin reactions. In particular, the most common reactions are localized eczematous eruptions at the injection sites, which may be secondarily generalized. Rare systemic reactions have been reported. We report a challenging case of eczema-like plaques secondary to enoxaparin, misdiagnosed as DRESS syndrome.

\section{CASE REPORT}

A 60-year-old female patient with a past medical history of mitral valve replacement under anticoagulant treatment (Sintrom $\left.{ }^{\circledR}\right)$, hypertension under Detensiel, and a frontal hemorrhagic stroke with seizures 2 months ago consulted our department for a sudden onset of a generalized pruritic rash. On interrogatory, she stated that she had been treated with carbamazepine (Tegretol) and "enoxaparin" type heparin for 2 months, but the heparin was discontinued 4 days before the onset of clinical manifestations. All other medications were taken regularly for 4 years. On examination, she was apyretic with a good general condition. There was facial edema, cheilitis, and a rash made of eczematous lesions. These lesions were electively located on the abdomen, lateral faces of the thighs, and arms at the sites of previously ecchymotic lesions that corresponded to the sites of enoxaparin injections (Figs. 1 and 2). There was no palpable peripheral lymphadenopathy. Blood analysis showed eosinophilia $=2500 \mathrm{e} / \mathrm{mm}^{3}$, lymphopenia $=780$ e $\mathrm{mm} 3$, C-reactive protein $=33$, $\mathrm{LDH}=347$, gamma GT $=526(10 * \mathrm{~N})$ and lipasemia $=$ $126\left(2.5^{*} \mathrm{~N}\right)$. Previous blood tests were within normal limits; gamma GT and lipase abnormalities were a new finding for our patient. Serological tests for hepatitis (A, B and $\mathrm{C}$ ), cytomegalovirus and human immunodeficiency virus were negative, as were autoantibody tests. Initially,

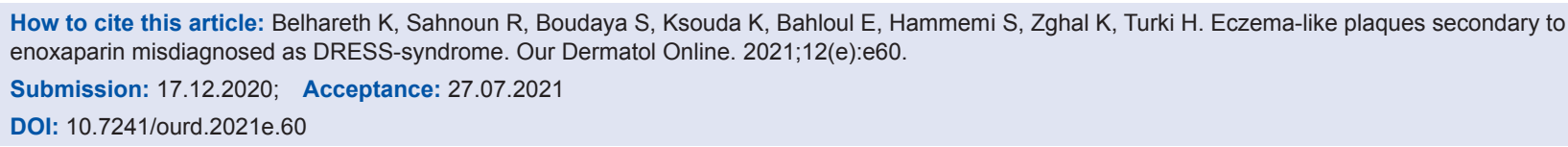


Tegretol was discontinued and a skin biopsy was performed. It showed a spongiotic reaction pattern with superficial perivascular infiltrate with lymphocytes, histiocytes and some eosinophils. There were no necrotic keratinocytes and direct immunofluorescence was negative. Treatment with topical corticosteroid therapy resulted in rapid improvement of the lesions within 7 days. After one month of treatment, laboratory data were within normal limits. A patch test with Tegretol was negative. Finally, we maintained the diagnosis of generalized eczema to heparin. To identify an alternative heparin and after obtaining informed consent, prick tests and intradermal tests were performed with a panel of unfractionated heparin (UFH) and LMWHs. Intradermal tests were read at $20 \mathrm{mn}, 2$ days and 6 days. All prick tests were negative. Intradermal tests with nadroparin, tinzaparin, Sodic Heparin, enoxaparin, and calciparin were positive at 48 hours (Figs. 3 and 4). The patient was seen at 3-month follow-up, laboratory values were within normal limits, and there was no recurrence of the lesions.

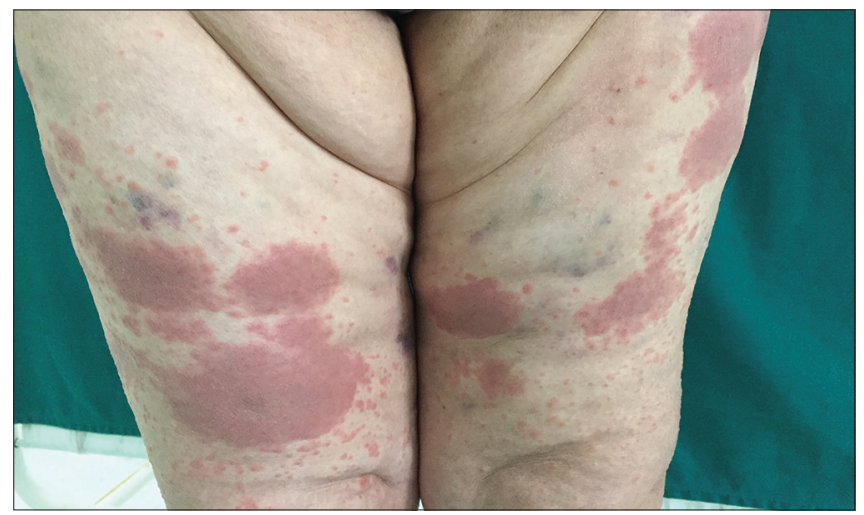

Figure 1: Eczematous lesions located on the lateral faces of the thighs at the site of enoxaparin injections.

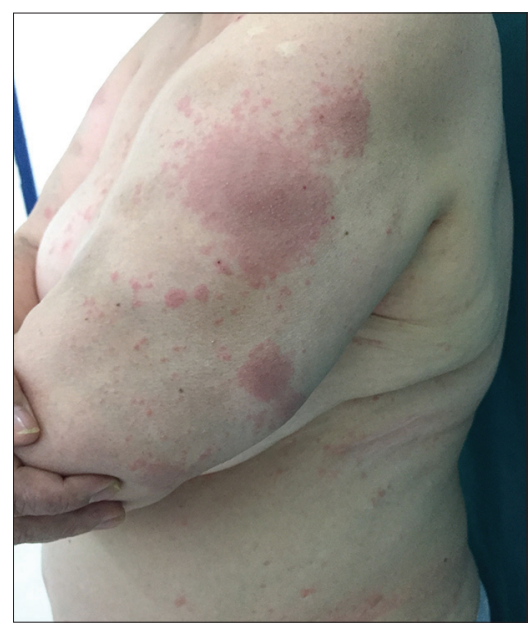

Figure 2: Eczematous lesions located on the lateral faces of the arms at the site of enoxaparin injections.

\section{DISCUSSION}

We present a noteworthy case of eczema-like plaques secondary to enoxaparin. Initially, we suspected the diagnosis of tegretol-induced DRESS syndrome based on tegretol uptake, facial edema, cheilitis, and visceral involvement. DRESS is a severe drug reaction characterized by fever, rash, eosinophilia, and one or more organ failures occurring 1-8 weeks after drug initiation. According to a recently published RegiSCAR score, DRESS is considered possible in our case (score $=3$ ), but patch test for Tegretol was negative and biopsy was against this diagnosis [1]. Regarding the Enoxa-induced DRESS syndrome, the biopsy was against it. To our knowledge, only one case of heparin-induced DRESS syndrome has been reported in the literature [2].

The final diagnosis was generalized eczema to heparin given the exclusive localization at the

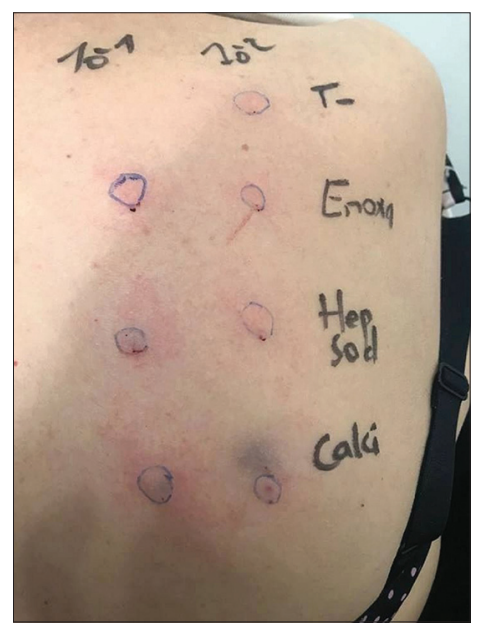

Figure 3: Positivity of intradermal tests with enoxaparin, Sodic heparin and calciparin at 48 hours.

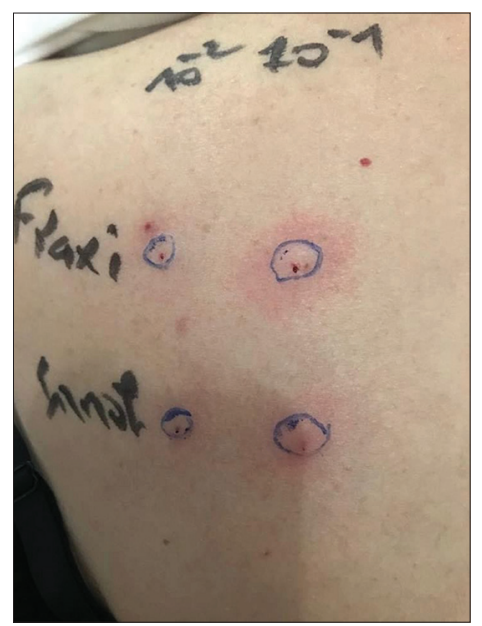

Figure 4: Positivity of intradermal tests with nadroparin, tinzaparin at 48 hours. 
sites of enoxaparin injections, the result of the biopsy and the rapid resolution of the eruption. Heparin hypersensitivity is a rare and unknown phenomenon; the first case was described in 1988 [3]. The physiopathologic mechanism of this reaction is binding of heparin to dermal proteins, which triggers a IV type hypersensitivity reaction [4]. The risk factors for this reaction are female gender, obesity, pregnancy and prolonged heparin treatment [5]. Clinically, it presents with eczematiform lesions at heparin injection sites with a delay of two days to two weeks or even months. To our knowledge, no case of eczema has been reported after discontinuation of heparin treatment. This rare occurrence could be explained by the persistence of heparins in subcutaneous adipose tissue, especially in infiltrated lesions. Cases with generalized rash or systemic eczema have been reported [6,7]. Differential diagnoses are numerous: sensitization to antiseptics, needle nickel and preservatives (in heparins), heparin necrosis (heparin-induced thrombocytopenia), urticaria-like immediate reactions, vasculitis and Fixed Pigmented Erythema. The allergological investigation is essentially based on intradermal reactions, which are necessary to confirm heparin sensitization and to identify alternative molecules that can be reintroduced. Indeed, polysensitization is possible with crossreactions between the different types of unfractionated heparins, low molecular weight heparins and synthetic heparinoids, as in our case. Intravenous fondaparinux, a new chemically synthesized selective inhibitor of activated factor $\mathrm{X}$, and heparin would be a possible alternative because of the lower risk of reactivity. However, rare cross-reactions between fondaparinux and heparins have been described.

\section{CONCLUSION}

In summary, heparins are commonly used in medical and surgical practice. It is important to know the possibility of various hypersensitivity reactions with this class of substances. The dermatologic effects of heparins are numerous with clinical pictures that can be misleading.

\section{Consent}

The examination of the patient was conducted according to the principles of the Declaration of Helsinki.

The authors certify that they have obtained all appropriate patient consent forms, in which the patients gave their consent for images and other clinical information to be included in the journal. The patients understand that their names and initials will not be published and due effort will be made to conceal their identity, but that anonymity cannot be guaranteed.

\section{REFERENCES}

1. Kardaun SH, Sekula P, Valeyrie-Allanore L, Liss Y, Chu CY, Creamer D, Sidoroff A, Naldi L, Mockenhaupt M, Roujeau JC; RegiSCAR study group. Drug reaction with eosinophilia and systemic symptoms (DRESS): an original multisystem adverse drug reaction. Results from the prospective RegiSCAR study. Br J Dermatol. 2013;169:1071-80.

2. Ronceray S, Dinulescu M, Le Gall F, Polard E, Dupuy A, Adamski H. Enoxaparin-Induced DRESS Syndrome. Case Rep Dermatol. 2012;4:233-7.

3. Klein GF, Kofler H, Wolf H, Fritsch PO. Eczema-like, erythematous, infiltrated plaques: a common side effect of subcutaneous heparin therapy. J Am Acad Dermatol. 1989;21:703-7.

4. Dabas G, De D, Handa S, Aggarwal D. Delayed-type hypersensitivity reaction to enoxaparin in a pregnant woman. Postgrad Med J. 2019;95:506.

5. Wutschert R, Piletta P, Bounameaux H. Adverse skin reactions to low molecular weight heparins: frequency, management and prevention. Drug Saf. 1999;20:515-25.

6. Komericki P, Grims R, Kränke B, Aberer W. Acute generalized exanthematous pustulosis from dalteparin. J Am Acad Dermatol. 2007; 57:718-21.

7. Govind B, Gnass E, Merli G, Eraso L. Hemorrhagic bullous dermatosis: a rare heparin-induced cutaneous manifestation. Hosp Pract (1995). 2016;44:103-7.

Copyright by Kamar Belhareth, et al. This is an open access article distributed under the terms of the Creative Commons Attribution License, which permits unrestricted use, distribution, and reproduction in any medium, provided the original author and source are credited.

Source of Support: Nil, Conflict of Interest: None declared. 